\title{
A Case of Subacute Ataxia in the Summertime: Tick Paralysis
}

\author{
Christin B. Laufer, $M D^{7}$ and Nicole Chiota-McCollum, $M D^{2}$ \\ 'Department of Internal Medicine, Keesler Medical Center, Biloxi, MS, USA; ²Department of Neurology, Keesler Medical Center, Biloxi, MS, USA.
}

Tick paralysis is caused by a neurotoxin secreted in the saliva of a gravid female tick, and manifests with ataxia, areflexia, ascending paralysis, bulbar palsy, and ophthalmoparesis. An 84-year-old man presented in June in coastal Mississippi with several days of subacute ataxia, bulbar palsy, unilateral weakness, and absent deep tendon reflexes. MRI/MRA and extensive serum and cerebrospinal fluid investigations were unrevealing. His symptoms progressed over several days, until his nurse discovered and removed an engorged tick from his gluteal fold. Within hours of tick removal, his subacute symptoms completely resolved. While tick paralysis is rare in adults, it is a condition that internists should be familiar with, particularly in seasons and areas with high prevalence of disease. This case also highlights the importance of performing a thorough skin exam on patients with the aforementioned neurologic abnormalities.

KEY WORDS: Infectious disease; Neurology; Neuromuscular disorder; Clinical vignette.

$\mathrm{J}$ Gen Intern Med 30(8):1225-7

DOI: $10.1007 / \mathrm{s} 11606-015-3276-8$

(c) Society of General Internal Medicine 2015

\section{INTRODUCTION}

Tick paralysis is a rare condition caused by the secretion of a neurotoxin in the saliva of a gravid female tick. ${ }^{1,2}$ It manifests with ascending paralysis, ataxia, areflexia, bulbar symptoms (dysphagia, dysphonia, dysarthria), and ophthalmoparesis (weakness of the extraocular muscles) after several days of tick attachment. ${ }^{1}$ Peak incidence is in the summertime, when the tick emerges from hibernation. ${ }^{1}$ In contrast to pediatric populations, which are typically affected, adults are rarely affected, as the neurotoxin concentration is thought to be attenuated by the larger adult body mass. ${ }^{3}$ Without tick removal, the disease can quickly become life-threatening, compromising respiratory muscle strength and resulting in respiratory failure. If missed, tick paralysis can portend significant morbidity and mortality. ${ }^{2}$ The hallmark feature of tick paralysis is a dramatic resolution of symptoms with one of the easiest treatments available- tick removal. $^{4}$

Received August 19, 2014

Accepted February 27, 2015

Published online March 21, 2015

\section{CASE PRESENTATION}

An 84-year-old man presented in June in coastal Mississippi with two days of unsteady gait and slurred speech. He was oriented to person, place, time, and situation, and did not have any memory loss. He had a past medical history of hypothyroidism, chronic kidney disease stage 3, and hyperlipidemia, but no prior history of stroke or alcoholism. At baseline, he reported having a normal gait and performance status. There were no antecedent illnesses, vaccinations, travel, new medications, known insect bites, or alcohol binges. Social history revealed that he was an avid outdoorsman in a rural community and that he consumed 1-2 drinks per week but had no history of heavy alcohol use. On exam, the patient was a wellgroomed man whose vital signs were within normal limits and whose body mass index was 25 . On neurologic exam, he was alert and oriented to person, place, time, and situation. His speech was slurred, but with normal comprehension. Cranial nerve exam revealed bilateral ophthalmoparesis, evidenced by weakness in his extraocular movements. Motor exam revealed bilateral upper extremity pronator drift, with the left arm more prominent than the right. His muscle strength was $4 / 5$ in his deltoids, triceps and hip flexors bilaterally. His reflexes were absent bilaterally. His sensation to light touch and proprioception were intact bilaterally. Cerebellar exam revealed dysmetria in his upper and lower extremities, and he was unable to perform rapid alternating movements. On gait examination, he demonstrated a wide-based gait with marked truncal ataxia with standing and sitting. Further maneuvers were deferred due to his high risk of falls. The patient's symptoms progressed over the following 48 hours, resulting in an inability to stand or even sit unassisted due to his profound ataxia.

Basic lab work, including a complete blood count and complete metabolic panel, yielded results within normal limits. Other serum tests included erythrocyte sedimentation rate, Creactive protein, hemoglobin $\mathrm{A} 1 \mathrm{C}$, thyroid-stimulating hormone, vitamin B12, serology for Borrelia burgdorferi, vitamin E levels, copper level, heavy metal levels, and rapid plasma regain (Table 1), all of which were unremarkable. The results of a urine drug screen were negative. Cerebrospinal fluid examination revealed the absence of an albuminocytologic dissociation, negative cultures, a non-reactive venereal disease research laboratory (VDRL), unremarkable West Nile virus serology, and no evidence of antibodies associated with paraneoplastic cerebellar degeneration (Table 2). A non-contrast CT of the head revealed results within normal limits. An MRI of the brain 
Table 1 Serum Studies

\begin{tabular}{lll}
\hline \hline Lab test & Admission value & Reference range \\
\hline RPR & Non-reactive & Non-reactive \\
Vitamin B12 $(\mathrm{pg} / \mathrm{mL})$ & 1,262 & $211-946$ \\
Arsenic $(\mathrm{mcg} / \mathrm{L})$ & $<2$ & $0-2$ \\
Mercury $(\mathrm{mcg} / \mathrm{L})$ & $<2$ & $0-2$ \\
Lead $(\mathrm{mcg} / \mathrm{dL})$ & $<10$ & $0-10$ \\
A-Tocopherol $(\mathrm{mg} / \mathrm{L})$ & 9.6 & $5.7-19.9$ \\
\hline
\end{tabular}

$R P R$ rapid plasma regain, Tocopherol Vitamin E

and MR angiogram of the head and neck were obtained after 48 hours of hospital admission, and ruled out an acute ischemic event or other explanation of his subacute symptomatology.

Our initial differential diagnosis included an acute ischemic event, Guillain-Barré syndrome, the Miller Fisher variant of Guillain-Barré syndrome, vitamin B12 deficiency, Wernicke's encephalopathy due to thiamine deficiency, vitamin E deficiency, heavy metal toxicity, West Nile virus infection, tabes dorsalis, early disseminated Lyme disease, and paraneoplastic cerebellar degeneration. Although the patient denied any history of alcoholism, given his symptoms of ophthalmoparesis and ataxia, parenteral thiamine was administered during his hospitalization in an attempt to reverse a possible underlying Wernicke's encephalopathy.

Over the first several hospital days, the patient's symptomatology progressed rapidly despite empiric treatment with intravenous thiamine. On the fourth hospital day, his nurse discovered and removed an engorged tick from the his gluteal fold. The tick was determined to be Dermacentor variabilis based on its morphology and epidemiology in the Gulf Coast area. Within hours of tick removal, the patient demonstrated a remarkable improvement, with complete recovery of his deep tendon reflexes, normal extraocular movements and muscle strength, resolution of his truncal and appendicular ataxia, and

Table 2 Cerebrospinal Fluid Studies

\begin{tabular}{lll}
\hline \hline Lab Test & Admission value & Reference range \\
\hline CSF color & Colorless & Colorless \\
CSF WBC (per mcL) & 2 & $0-5$ \\
CSF RBC (per mcL) & 0 & 0 \\
CSF Glucose (mg/dL) & 66 & $40-70$ \\
CSF Protein (mg/dL) & 46 & $15-45$ \\
CSF Culture & No growth & No growth \\
CSF VDRL & Non-reactive & Non-reactive \\
CSF WNV IgG & $<1.3$ & $<1.3$ \\
CSF WNV IgM & $<.9$ & $<.9$ \\
ANNA-1 & Negative & Negative \\
ANNA-2 & Negative & Negative \\
ANNA-3 & Negative & Negative \\
AGNA-1 & Negative & Negative \\
PCA-1 & Negative & Negative \\
PCA-2 & Negative & Negative \\
PCA-Tr & Negative & Negative \\
CRMP & Negative & Negative \\
\hline
\end{tabular}

$W B C$ white blood count, $R B C$ red blood count, VDRL venereal disease research laboratory, WNV West Nile virus, ANNA anti-neuronal nuclear antibody (Anti-Hu), AGNA anti-glial nuclear antibody, PCA purkinje cell cytoplasmic antibody (Anti-Yo), CRMP collapsin response mediator protein 5. recovery of his gait. By hospital discharge, he had completely returned to his baseline level of neurologic function. To date, he has remained well and asymptomatic.

\section{DISCUSSION}

The temporal relationship between tick removal and the patient's recovery are characteristic of tick paralysis. ${ }^{4}$ The tick that was removed was presumed to be Dermacentor variabilis, based on its morphology and epidemiology. Culprit ticks reported in the United States include Dermacentor variabilis, which is widespread east of the Rocky Mountains and in limited areas of the Pacific coast, and Dermacentor andersoni, which is found in the Rocky Mountain states. ${ }^{1,5}$ The culprit in this case was thought to be an adult female, as ticks in the larval and nymphal stages do not bite humans. ${ }^{5}$ The vast majority of tick paralysis cases occur in children; adults are very seldom affected. The most common demographic in the United States to experience tick paralysis is female patients under 8 years of age who have long hair, presumably because the tick can be difficult to identify on the scalp. ${ }^{5}$

While an alternative explanation for the patient's presentation is Wernicke's encephalopathy, which can present with confusion, ataxia, ophthalmoparesis, and nystagmus, he did not endorse confusion or nystagmus, and denied any heavy alcohol consumption. In addition, symptom improvement would be expected after 2 days of parenteral thiamine administration, which was not demonstrated in this case. ${ }^{6}$ Moreover, the patient's MRI did not demonstrate any atypical lesions or enhancement in the mammillary bodies or thalamus, which, if present, would be suggestive of Wernicke's encephalopathy. ${ }^{6}$ Furthermore, there was no temporal relationship between thiamine administration and the patient's improvement.

This is a unique case of a rare disease that affects adults infrequently. ${ }^{1}$ Although tick bites are often painless, it is unusual that the tick persisted in the gluteal fold of our patient, who was not obese. Tick paralysis should be included in the differential diagnosis in any patient who presents in the summertime with subacute ascending paralysis, areflexia, ataxia, bulbar palsy, or ophthalmoparesis. A thorough skin exam should be performed on any patient whose differential diagnosis includes tick paralysis, as failure to do so could portend an ominous prognosis.

Acknowledgments: There were no funding sources, grants, or other financial support. This case was presented at the national American College of Physicians meeting in April 2014.

Conflict of Interest: The authors declare that they do not have a conflict of interest.

Corresponding Author: Christin B. Laufer, MD; Department of Internal MedicineKeesler Medical Center, Biloxi, MS 39534, USA (e-mail: christin.b.laufer@gmail.com). 


\section{REFERENCES}

1. Ropper A, Samuels M. Adam's and Victor's Principles of Neurology: Tick Paralysis. 9th ed. New York: McGraw-Hill; 2009.

2. Pecina C. Tick paralysis. Semin Neurol. 2012;32(5):531-2.

3. Felz M, Smith C, Swift T. A six-year-old girl with tick paralysis. N Engl J Med. 2000;342:90-4.
4. Vedenarayanan V, Sorey WH, Subramony SH. Tick paralysis. Semin Neurol. 2004;24(2):181-4.

5. Centers for Disease Control and Prevention - Ticks. http://www.cdc.gov/ ticks/. February 27, 2015.

6. Galvin R, Brathen G, Ivashynka A, et al. EFNS guidelines for diagnosis, therapy and prevention of Wernicke encephalopathy. Eur $\mathrm{J}$ Neurol. 2010;17:1408-18. 